\title{
Development of a sky imaging system for short-term solar power forecasting
}

\author{
B. Urquhart, B. Kurtz, E. Dahlin, M. Ghonima, J. E. Shields, and J. Kleissl \\ University of California, San Diego, California, USA \\ Correspondence to: B. Urquhart (burquhar@ucsd.edu)
}

Received: 27 February 2014 - Published in Atmos. Meas. Tech. Discuss.: 19 May 2014

Revised: 29 October 2014 - Accepted: 9 January 2015 - Published: 20 February 2015

\begin{abstract}
To facilitate the development of solar power forecasting algorithms based on ground-based visible wavelength remote sensing, we have developed a high dynamic range (HDR) camera system capable of providing hemispherical sky imagery from the circumsolar region to the horizon at a high spatial, temporal, and radiometric resolution. The University of California, San Diego Sky Imager (USI) captures multispectral, 16 bit, HDR images as fast as every $1.3 \mathrm{~s}$. This article discusses the system design and operation in detail, provides a characterization of the system dark response and photoresponse linearity, and presents a method to evaluate noise in high dynamic range imagery. The system is shown to have a radiometrically linear response to within $5 \%$ in a designated operating region of the sensor. Noise for HDR imagery is shown to be very close to the fundamental shot noise limit. The complication of directly imaging the sun and the impact on solar power forecasting is also discussed. The USI has performed reliably in a hot, dry environment, a tropical coastal location, several temperate coastal locations, and in the great plains of the United States.
\end{abstract}

\section{Introduction}

Solar power output of an individual generator, or even a fleet of generators, will have some level of variability due to the nature of the input source of light from the sun. The source of short-term variability of irradiance at the earth's surface is clouds and atmospheric particulates, which are generally not controllable. To reliably integrate increasing amounts of solar power into the electric grid, forecasting, storage, additional transmission, and ancillary power generation services will constitute a portfolio of solutions to counteract variability.

From a planning and operations perspective, grid operators require consumption and generation estimates from years to minutes ahead. On the scale of days to minutes, solar power output forecasts are provided by some combination of weather models and remote sensing of clouds, along with stochastic learning methods. Forecasting of solar radiation in the $0-30 \mathrm{~min}$ ahead time frame poses unique challenges. High resolution models reported for both satellite and numerical weather prediction can issue forecasts that have $5 \mathrm{~min}$ time steps for a one kilometer grid (Mathiesen et al., 2013; Perez et al., 2013), while the best operational models often have coarser resolutions in both space and time (Dupree et al., 2009; Rogers et al., 2012; Mathiesen and Kleissl, 2011). However, in numerical models, timing and/or positioning errors of clouds are inevitable, and for satellites, infrequent image capture and parallax effects can result in inaccurate georeferencing of clouds. These errors make it difficult to achieve an accurate, high resolution short term solar forecast. This motivates a need for other forecasting tools and observational methods.

One short-term forecasting technology that has emerged recently has been based on remote sensing of clouds from ground-based imaging systems (Chow et al., 2011; Marquez and Coimbra, 2013; Urquhart et al., 2013; Yang et al., 2014; Fu and Cheng, 2013). Urquhart et al. (2013) applied the forecasting method to $48 \mathrm{MW}$ of photovoltaic generation. One of the key conclusions from that work was that the Total Sky Imager (TSI), while providing the ability to monitor sky conditions, had shortcomings that limit its effectiveness for solar power forecasting. 
This work describes the development of sky imaging hardware for short-term solar power forecasting at UCSD. The UCSD Sky Imager (USI) provides unique capabilities needed for forecasting research and applications. The goal of this article is to provide details on the USI system, and report on its imaging performance so that other workers in this field may make more informed purchasing and design decisions. The remainder of Sect. 1 discusses hardware requirements for sky imaging, and reviews relevant sky imaging hardware presented in the literature. Specifics of the USI hardware development and system operations are covered in Sects. 2 and 3 , respectively. The imaging performance of the USI is characterized in Sect. 4, and Sect. 5 presents deployments of the USI to date.

\subsection{Imaging system considerations for solar forecasting}

Sky imagers were historically built for recording meteorological conditions such as sky cover. For this purpose it is not critical to image the area directly around the sun, so many systems have sun occluding devices to prevent direct sunlight from entering the optics. When the sun is unobstructed, more than $90 \%$ of the photons entering the optics can come from the direct solar beam. For most camera systems, the handful of pixels encompassing the sun saturate and thus directbeam signal intensity is only known to exceed the saturation threshold. Immediately outside the direct beam is a region of intense forward scattering. Aerosols and dust scatter the direct beam predominantly in the forward direction, increasing the size of region around the sun that will potentially saturate in a sky image. Cloud droplets and ice crystals, when present, also predominantly scatter in the forward direction and, depending on the scattered intensity reaching the camera, can further extend the size of the region that will saturate. Obtaining on scale image information about the region around the sun requires appropriate imaging hardware and methods, especially when the region around the sun has a very high intensity. Further, this high intensity region can cause image quality degradation through internal reflections, diffraction caused by the aperture, sensor saturation, smear and blooming, and, potentially, sensor damage (see Sect. 4.7 for further discussion relating to the USI). The significance of each of these potential issues is imaging system dependent.

The use of occluding devices eliminates many of these potential issues, which is why they are often adopted. Blocking the sun and the surrounding area, however, eliminates important sky condition information needed to provide reliable forecasts in the first few minutes $(<5 \mathrm{~min})$ of the forecast period. If the occluding device obstructed a minimal amount of the image along with precision-positioning mechanisms, it could then be used without adversely affecting immediateterm forecast accuracy. For cost and reliability reasons, these requirements are difficult to achieve in practice. The TSI, for example, has a shadow band that occludes $14 \%$ of the sky hemisphere, always in the region near the sun. Even for a
$1.3 \mathrm{~km}^{2}$ solar power plant, the shadow band on the TSI has been demonstrated to obscure sky condition information for over half of the plant (Urquhart et al., 2013). With proper design, and appropriate image capture and correction algorithms, sky imaging systems can acquire atmospheric information from an appreciable amount of the region around the sun (e.g., Stumpfel et al., 2004). To this end, the high dynamic range imaging method described in Sect. 4.5 provides a simple and robust approach.

The spectral content of the sky scene provides important information for the remote sensing of clouds and water vapor. Most camera systems capture visible wavelength imagery that spans between 350 and $800 \mathrm{~nm}$. This allows the measurement of shortwave solar radiation that is scattered by the clouds, atmospheric gases and aerosols. Silicon-based image sensors used in visible wavelength cameras are also sensitive up to $1.1 \mu \mathrm{m}$ in the near infrared. The sixteen bit (or higher) versions of these, with a set of selectable bandpass and neutral density filters, can be used for enhanced day and nighttime cloud detection (Shields et al., 2013). Longwave infrared (LWIR) imaging in the $8-13 \mu \mathrm{m}$ range measures cloud brightness temperatures which can be used to segment different cloud layers, estimate cloud heights, and potentially determine optical depth. LWIR imaging hardware costs significantly more than common visible wavelength imaging hardware, and it may not be practical for widespread deployment.

The image formation process in a sky imaging camera redirects radiant energy from the sky hemisphere onto the two dimensional image plane. Geometric and radiometric calibrations turn the brightness information at a given pixel position into a measurement of sky radiance at a given look angle. Geometric calibration relates a pixel position on the sensor array to a set of angles (azimuth and zenith) in a defined world coordinate system. This is a necessary step to accurately geolocate clouds. Solar forecasting methods such as Chow et al. (2011) and Yang et al. (2014) require geometric calibration of the imager because cloud positions are explicitly computed. Time of arrival methods such as Marquez and Coimbra (2013) or Wood-Bradley et al. (2012) do not require geometric calibration because only a forecast of when a cloud will occlude the sun for the location of the imager is needed. A treatment of geometric camera calibration is beyond the scope of this article, but the interested reader is referred to Faugeras (1993) or Hartley and Zisserman (2004) for an introduction to the calibration process, and Urquhart et al. (2015) for more on the geometric calibration of the USI. Radiometric calibration makes it possible to determine the radiance of the scattered light coming from a portion of the sky (Shields et al., 1998a; Feister and Shields, 2005; Román et al., 2012), and can be used as input to retrieval algorithms for a number of optical properties of atmospheric aerosols that impact solar energy generation (Nakajima et al., 1996).

The spatial resolution of the camera (i.e., the pixel density) directly impacts the cloud resolving ability of the camera. 
The size (i.e., area) of a cloud element $A_{\text {cld }}\left(\mathrm{m}^{2}\right)$ observed by a single pixel at a cloud height $H$ can be described as

$A_{\text {cld }}=\pi H^{2} \sec ^{2}(\theta) \tan ^{2}(\alpha)$,

where $\theta$ is the angle from the optical axis, and $\alpha$ defines the field of view of the pixel. It has been assumed for simplicity that the field of view is a circular cone with $\alpha$ representing the cone half-angle, with the cloud element orthogonal to the cone axis The solid angle subtended by the cone, and thus the pixel, is then $2 \pi(1-\cos \alpha)$. For an equisolid angle lens (Sect. 2.1) $\alpha$ is nominally constant over the entire field of view, and $A_{\text {cld }}$ is a function of $\theta$ only. If the patch of cloud is projected to a plane parallel to the ground (Chow et al., 2011; Urquhart et al., 2013; Yang et al., 2014), for small $\alpha$ the area of the patch can be approximated as

$S_{\text {cld }}=\frac{1}{2} \pi H^{2} \sec (\theta) \tan (\alpha)(\tan (\theta+\alpha)-\tan (\theta-\alpha))$.

If this cloud element is projected onto the surface as a "cloud shadow", the area is approximately $S_{\text {cld }}$ when ignoring effects due to terrain. For an equisolid angle lens, the value of $\alpha$ is related to the pixel density as

$\alpha=\operatorname{acos}\left(1-1 / n_{\text {pix }}\right)$,

where $n_{\text {pix }}$ is the number of pixels occupying the image of the sky (assumed to be a $2 \pi$ steradian hemisphere). As $n_{\text {pix }}$ increases, $\alpha$ decreases, and thus the areas $A_{\text {cld }}$ and $S_{\text {cld }}$ decrease, i.e., the resolving power of the system increases (assuming the lens is not the limiting factor). At $\theta=30^{\circ}, A_{\text {cld }}$ for the TSI ( $n_{\text {pix }} \approx 0.14$ Mpixels) is $544 \mathrm{~m}^{2}$ at a $3 \mathrm{~km}$ cloud height, whereas for the USI ( $n_{\text {pix }} \approx 2.32$ Mpixels) it is $33 \mathrm{~m}^{2}$. For $S_{\text {cld }}$, the corresponding values are 1257 and $75 \mathrm{~m}^{2}$, respectively. If a pixel is incorrectly labeled a cloud (or as clear), it has a much larger areal impact for the TSI than for the USI.

\subsection{Existing sky imaging hardware}

There are three fields where a majority of sky imaging work has been performed: atmospheric sciences, forestry and ecology, and astronomy. Camera developers in astronomy are typically concerned with having a high sensitivity and low noise so that a high percentage of incoming photons from stars, asteroids and other faint objects are converted into charge carriers on the image sensor. The sensors used are often full frame charge-coupled devices (CCDs) because of the high quantum efficiency and fill factor, but require mechanical shuttering which limits the frame rate of the system. One system similar to the USI from the astronomy field is the All Sky Infrared Visible Analyzer (ASIVA, Klebe et al., 2014; Sebag et al., 2008) with a dual camera system that captures both visible and LWIR images. It is one of the few LWIR dioptric (refraction-based) whole-sky designs (catadioptric (reflection and refraction) designs similar to the TSI are more common). It uses a $640 \times 512$ uncooled microbolometer array sensitive in the $8-13 \mu \mathrm{m}$ range with a germanium fisheye lens. The system has an 8-slot filter wheel allowing for multiband LWIR measurements. The ASIVA also has a highresolution visible camera with an 8-slot filter wheel (specific camera model has varied by ASIVA unit).

The area of forestry has extensively used hemispherical photography (Brown, 1962; Anderson, 1964). The highdynamic-range all-sky-imaging system (HDR-ASIS) is a CMOS-based camera that leverages multiple exposures to create a high-dynamic-range (HDR) composite sky image for ecosystem and canopy research (Dye, 2012).

Researchers in atmospheric science have very actively developed their own instruments over the years. In fact, Hill (1924) mentions cloud photography as a motivation in developing the first true fisheye lens design. Digital sky photography began in the 1980s with the development of personal computers, and one of the leading groups developing imaging systems for atmospheric observation was the Atmospheric Optics Group at the Scripps Institute of Oceanography's (SIO's) Marine Physical Laboratory (Johnson et al., 1988, 1989). Their well known Whole Sky Imager (WSI) is still to this day one of the highest quality, if not the highest quality, sky imaging systems ever developed (Shields et al., 1998b, 2013). It was developed primarily for US military applications in the 1980s and early 1990s. More recent designs of the system had a $512 \times 512$-pixel temperature-controlled, 16-bit low-noise monochrome CCD camera. It used a Nikon Nikkor $8 f / 2.8(8 \mathrm{~mm}$ ) fisheye lens (equidistant projection, Sect. 2.1) and two filter wheels holding neutral density and spectral filters at multiple wavelengths. The image plane was the surface of a tapered fiber-optic bundle that interfaced directly to the CCD. Multiple corrections were made to the instrument to improve measurement quality: dark field correction; flat field correction (among other things, this corrected imaging issues caused by fiber optic imperfections); exposure corrections; linearity corrections; roll-off corrections; geometric calibration; and in some cases absolute radiometric calibration. By adjusting the neutral density and spectral filter selections, and/or the exposure time, the system achieved a wide dynamic range and could capture both daytime and nighttime imagery with high accuracy. The cloud detection algorithms developed over several decades were sophisticated, with accurate detection of haze, thin cloud, and opaque cloud (Shields et al., 1993a, b, 1998a; Feister and Shields, 2005).

The most widely used outdoor hemispheric camera system, first described by Long and DeLuisi (1998) as the Hemispheric Sky Imager, is the Total Sky Imager. It has been commercially available by Yankee Environmental Systems (YES) for over a decade, and has a proven track record of reliably recording sky conditions. The catadioptric optical design uses a spherical mirror to reflect the sky hemisphere into a downward-pointing camera. The system has relatively low spatial and radiometric resolution $(640 \times 480$ pixels, 


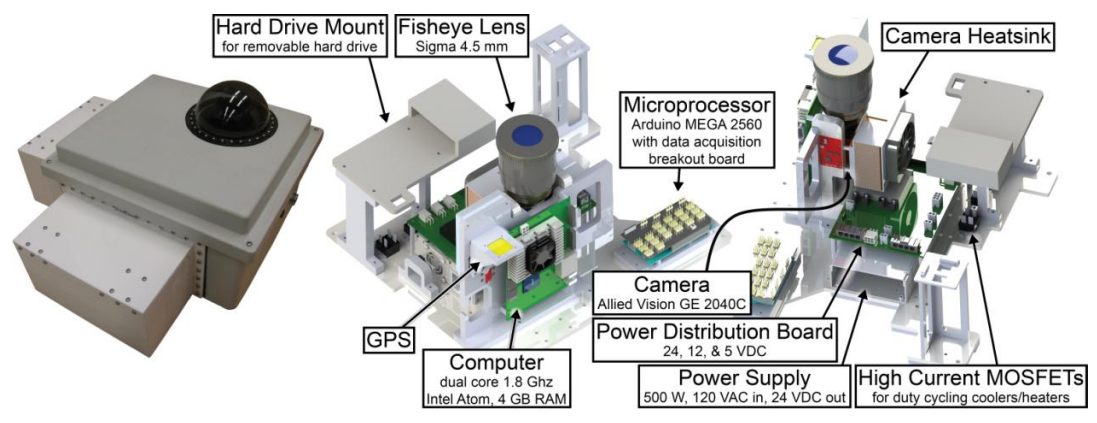

Figure 1. The UCSD Sky Imager enclosure (left) and a CAD model showing the layout of system components (right).

8 bits), and there is little control of the camera capture settings. An antireflective black rubber strip ("shadow band") affixed to the mirror prevents direct sunlight from reflecting into the camera optics which improves image quality and avoids damage to the sensor. The shadow band covers approximately 0.70 steradians of the hemisphere, which is about $14 \%$ of the image region used for forecasting $\left(<80^{\circ}\right.$ zenith angle). Accurate geometric calibration of the TSI is challenging because of the mirror design. Translation and rotation of the mirror with respect to the camera body (the camera is understandably not perfectly over the mirror center) makes modeling the camera geometry more complicated and thus calibration is more challenging than for upward pointing systems with a single lens. Additionally, the mirror is often slightly warped in shape (i.e., not perfectly spherical), and the surface is covered in small scale imperfections that produce local distortion. A comparison of the solar forecasting performance between the TSI and USI was performed by Gohari et al. (2014).

Beyond the WSI and TSI, a number of other imaging systems have been developed for atmospheric studies. A description of several of these can be found in Urquhart et al. (2013) and in Table 1. Outside of systems developed by research groups, there are alternatives to the TSI. The SONA (Sistema Automático de Observación de Nubes, Gonzales et al., 2012) uses a $1 / 3^{\prime \prime}, 640 \times 480 \mathrm{CCD}$, has integrated coolers, heaters and temperature sensors and is ruggedized for outdoor deployment. It has an integrated shadow band with azimuth control that shades part of the lens, but not the full optical system (i.e., it does not shade the entire dome). The Eko sky camera, built by Schreder, is reported to have 2 Mpixels, and like the SONA and TSI, has cloud detection software and a user interface. The Santa Barbara Instrument Group (SBIG) sells the Allsky-340C camera system based on a Truesense KAI- $0340,640 \times 480 \mathrm{CCD}$ with a specified dynamic range (defined Sect. 2.2) of up $69 \mathrm{~dB}$, and uses a $1.4 \mathrm{~mm}$ focal length Fujinon FE185C046HA-1 lens. The SBIG camera was used for solar forecasting research by $\mathrm{Fu}$ and Cheng (2013). Inclusion of a sky camera in this discussion is not meant to indicate it is not suitable for solar power forecasting. The list of systems noted here is far from com- prehensive, and with the potential of sky imagery for solar energy applications, new systems are continuously being developed.

\section{Hardware design and selection methods}

\subsection{Optical design}

The University of California, San Diego has developed its own sky imager (the USI, Fig. 1) to address the instrument needs for short term forecasting. The USI uses a Sigma $4.5 \mathrm{~mm}$ focal length fisheye lens which allows the entire image circle to fit on the sensor. This can easily be verified from the focal length, the lens projection, and sensor size. A conventional camera lens has the rectilinear projection function

$r_{s}=f \tan (\theta)$,

where $f$ is the focal length, $\theta$ is the angle from the optical axis, and $r_{s}$ is the distance from the principal point in the image plane. It is evident that this pinhole camera model cannot image points at $90^{\circ}$ from the optical axis with a sensor of finite size. In order to form the image of points that are $90^{\circ}$ from the optical axis within a finite image plane, distortion is required, and the type of distortion can be selected by the optical designer. The two most common projections used in fisheye lenses are the equidistant and equisolid angle projections, $r_{\mathrm{ed}}$ and $r_{\mathrm{es}}$, respectively:

$r_{\mathrm{ed}}=f \theta$

$r_{\mathrm{es}}=2 f \sin \left(\frac{\theta}{2}\right)$.

Each of these projection models provides different performance characteristics. The equidistant model provides a linear relation between incidence angle and distance from the principal point, and it has slightly less distortion at large angles from the optical axis than the equisolid angle projection. The equisolid angle projection is so-named because the solid angle subtended by a unit area on the image plane is constant, regardless of incidence angle (Miyamoto, 1964). A comparison of the different lens projections is shown in Fig. 2a, along 
Table 1. Research camera systems for sky atmospheric observations.

\begin{tabular}{|c|c|c|c|c|c|}
\hline System & Camera & Sensor & Resolution & Lens & Reference \\
\hline WSI & Photometrics S300 & $\mathrm{CCD}$ & $512 \times 512$ & Nikon, equidistant & Shields et al. (2013) \\
\hline WSC & - & $\mathrm{CCD}, 1 / 3^{\prime \prime}, 8.3 \mathrm{~mm}$ & $752 \times 582$ & $1.6-3.4 \mathrm{~mm}$ & Long et al. (2006) \\
\hline ASI (GFAT) & QImaging RETIGA 1300C & Sony ICX085AK CCD, 2/3", 12-bit & $1300 \times 1030$ & Fujinon FE185C057HA & $\begin{array}{l}\text { Cazorla et al. (2008) } \\
\text { Román et al. (2012) }\end{array}$ \\
\hline IFM-GEOMAR & - & 10-bit & $3648 \times 2736$ & - & Kalisch and Macke (2008) \\
\hline ASI (CAS) & - & $1 / 3^{\prime \prime} \mathrm{CCD}$ & $2272 \times 1704$ & equidistant & Huo and Lu (2012) \\
\hline
\end{tabular}
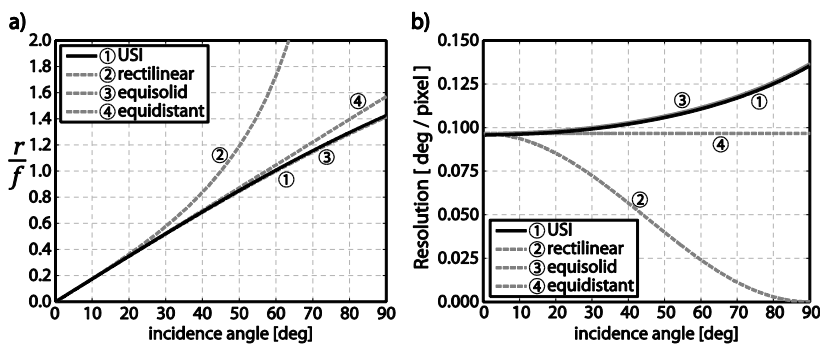

Figure 2. (a) Perspective (rectilinear), equidistant, and equisolid angle projection distances as a function of incidence angle, along with the projection for USI 1.2 determined from geometric calibration. The projection distance is normalized by the focal length. (b) Zenith angle resolution of projections in (a).

with that measured for the USI system. The angular resolution per pixel is shown in Fig. 2b. Figure $2 b$ assumes the sensor is $15.15 \mathrm{~mm}$ across containing 2048 pixels, and uses the specifications for USI 1.2 in Table 2. Even though the angular resolution at the horizon is coarser for an equisolid versus an equidistant projection at the same focal length, the former was selected for the USI because at large zenith angles, the horizontal configuration of clouds is difficult to determine because of self occlusion and perspective effects. Using more of the sensor area for the sky region overhead and near the sun (during midday) was preferred because these sky areas contain the clouds causing the current and near future solar power generation impacts when power output is highest.

For a given sensor size, the selected projection places a limit on the maximum allowable focal length of a lens while still being able to capture the complete sky dome (or conversely, the minimum sensor size given a focal length). The maximum allowable focal length for the equidistant projection $f_{\text {ed, max }}$ is $2 r_{\min } / \pi$ and for the equisolid angle projection $f_{\text {es, max }}$ is $r_{\text {min }} / \sqrt{2}$, where $r_{\text {min }}$ is the shortest distance from the principal point to the edge of the sensor. For the USI, with a sensor size of $15.15 \mathrm{~mm}, r_{\min }$ is $7.575 \mathrm{~mm}$ (assuming the principal point is in the center of the image sensor), and a focal length of less than $5.36 \mathrm{~mm}$ for the equisolid angle projection is required. Because the principal point will in general vary depending on machining and assembly tolerances of the components used, the value of $r_{\min }$ will vary. Table 2 shows the principal point location, $r_{\min }$, and $f_{\max }$, for several USI systems obtained from a nonlinear geomet-
Table 2. Intrinsic parameters and lens focal length selection parameters measured for 7 USI units. The principal point $\left(u_{o}, v_{o}\right)$ and focal length $f$ are measured for each USI. The minimum distance to the sensor edge $r_{\min }$ from $\left(u_{o}, v_{o}\right)$ yields the maximum allowable focal lengths $f_{\text {ed,max }}$ and $f_{\text {es, max }}$ for the equidistant and equisolid angle projections, respectively. Units are in mm, except for $u_{o}$ and $v_{o}$ which are given in pixels.

\begin{tabular}{lrrrrrr}
\hline USI no. & $u_{o}$ & $v_{o}$ & $f$ & $r_{\text {min }}$ & $f_{\text {ed,max }}$ & $f_{\text {es, max }}$ \\
\hline 1.1 & 1032 & 965 & 4.437 & 7.139 & 4.545 & 5.048 \\
1.2 & 1040 & 970 & 4.386 & 7.176 & 4.568 & 5.074 \\
1.5 & 1033 & 963 & 4.429 & 7.124 & 4.535 & 5.037 \\
1.6 & 1028 & 991 & 4.377 & 7.331 & 4.667 & 5.184 \\
1.8 & 1023 & 1043 & 4.448 & 7.434 & 4.733 & 5.257 \\
1.9 & 1045 & 976 & 4.474 & 7.220 & 4.596 & 5.105 \\
mean & 1033.5 & 984.7 & 4.425 & 7.237 & 4.607 & 5.118 \\
SD & 7.3 & 27.7 & 0.034 & 0.111 & 0.071 & 0.079 \\
\hline
\end{tabular}

ric calibration of extrinsic and intrinsic parameters that minimized the squared pixel error between actual sun position measurements and modeled sun position. The NREL solar position algorithm (Reda and Andreas, 2004) was used for modeled sun position input. The principal point shows significant variation because the mounting location of the lens fluctuates by as much as $0.31 \mathrm{~mm}$. As a result, the radial distance to the edge of the detector fluctuates, and along with it the maximum allowable focal length.

Proper selection of the aperture diameter is important to ensure an appropriate flux of radiant energy impinges on the sensor plane. If the flux is high, very short exposure times are required to obtain quality sky images. Because there is no mechanical shutter in the USI, the sensor is always exposed and limiting the incoming radiant flux is a way to extend sensor life. If the aperture diameter is small, exposure time must be increased, and motion blur of the clouds is possible. The Sigma lens comes with an iris diaphragm which was not used to avoid diffraction caused by the iris blades (e.g., Fig. 3e). To reduce the amount the incoming radiant flux without the iris diaphragm, two methods were tested: (1) a rear neutral density (ND) gelatin filter with a transmissivity of $0.1 \%$, and (2) a fixed circular aperture for which several diameters were tested. The aperture was placed between the 9th and 10th lens elements using the iris mounting assembly. Stray light and spectral effects of each approach are discussed in Sect. 4. Undesirable diffraction patterns were observed on the USI for 


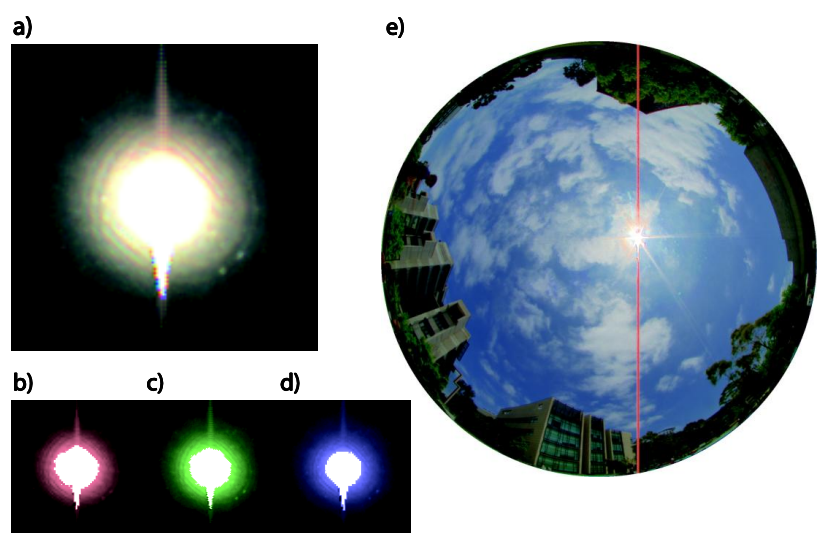

Figure 3. (a) Diffraction pattern measured with a $1000 \mu \mathrm{m}$ aperture on USI 1.8, with red, green, and blue color components shown in (b), (c), and (d), respectively. (e) Diffraction of the hexagonal iris blades in the stock lens.

circular apertures of diameter 300, 700, and $1000 \mu \mathrm{m}$ (Fig. 3). Because diffraction caused by a circular aperture generates a known Airy disk pattern, it is possible to partially correct the image with deconvolution processing, however this was not done in this work. To minimize the incoming flux while also minimizing diffraction, an aperture of $1250 \mu \mathrm{m}$ was selected. In comparison, the aperture diameter with the ND filter is $9520 \mu \mathrm{m}$. This large diameter noticeably reduces the depth of focus of the camera compared to the $1250 \mu \mathrm{m}$ aperture (depth of field is unaffected because a fisheye lens is used). The radiant flux is higher using an aperture of $1250 \mu \mathrm{m}$ compared to the ND filter configuration by a factor of 18 . This allows shorter exposures with less motion blur caused by longer integration times, but may also lead to increased sensor degradation in the long term due to the increased radiation on the sensor.

In order to develop a ruggedized system, it is necessary to protect the lens and properly seal the enclosure from the environment. For the lens to have full $180^{\circ}$ access to the sky with this requirement, a 1/16th inch thick, neutral density acrylic dome was used on the USI. The dome has a UV hardcoat applied to minimize transmission of high energy solar radiation which helps reduce component degradation. Amorphous silicate glass has superior transmissivity and scratch resistance than acrylic, but is more difficult to machine and handle, and designing proper sealing for a glass dome is more complicated (and thus more expensive). Polycarbonate, while having similar transparency and machining characteristics to acrylic, becomes opaque due to oxidation, making it a poor choice as a dome material (stabilizer additives can dramatically improve the lifetime). A drawback of acrylic is that it is susceptible to scratching from wind-blown particulates (common in the desert), improper cleaning, and birds which occasionally land on the dome and scratch it with their talons or beak. The use of a neutral density acrylic dome with a higher neutral density and an anti-reflective coating on the inner surface is being considered to improve image quality further.

\subsection{Camera and image sensor}

The USI uses an Allied Vision GE-2040C camera which contains a $15.15 \times 15.15 \mathrm{~mm}, 2048 \times 2048$ pixel Truesense KAI-04022 interline transfer CCD sensor. The camera is connected to the computer with a gigabit ethernet interface, and customized control is achieved by using the PvAPI for Linux provided by Allied Vision. For solar forecasting research, we have found that the ability to adjust exposure integration times, frame rates, regions-of-interest, and other parameters is a necessary capability.

The USI imaging system was designed to generate images suitable for cloud detection and motion processing. Cloud detection requires spectral measurements, and thus a spectral filtering method must be employed in some capacity. Coupled with a high quality sensor, camera, and lens, a mechanical shutter and color filter wheel can provide very high quality still spectral measurements. These moving components, however, complicate system design and HDR capture, and limit frame rates, therefore no mechanical shutter or color filter wheel were used. Spectral measurements were instead obtained by using a sensor with Bayer color filter array (CFA, Bayer, 1975).

The intensity range of the sky necessitates a sensor with a large dynamic range. Large dynamic range and global electronic shuttering are available from interline transfer CCDs, which is why this technology was selected for the USI. Dynamic range DR is defined by the ratio of maximum measurable signal to the noise floor:

$\mathrm{DR}=20 \log _{10} \frac{c_{\text {sat }}}{c_{\text {rd }}}$,

where $c_{\text {sat }}$ is the count value at saturation and $c_{\text {rd }}$ is the read noise. For a single USI exposure, $c_{\text {sat }}$ is 4095 counts and $c_{\text {rd }}$ is 3.8 counts. Read noise is introduced by the camera readout electronics, including output amplifiers and analog-todigital converters. For a single USI exposure, the dynamic range was measured to be $61 \mathrm{~dB}$ over the entire sensor. Testing by the camera manufacturer (following the EMVA 1288 standard) also gives a dynamic range of $61 \mathrm{~dB}$, along with a full well depth of $23600 \mathrm{e}^{-}$. The decrease in dynamic range from the $72 \mathrm{~dB}$ specified by the $\mathrm{CCD}$ manufacturer is due to an increase of sensor operating frequency. The output amplifiers limit the amount of charge that can be read per unit time, and thus frame rate has been traded for dynamic range. In this higher frame rate case, the dynamic range for the KAI-04022 is not limited by pixel charge capacity, because the $7.4 \mu \mathrm{m}$ pixels can hold nominally $40000 \mathrm{e}^{-}$. In an HDR image (Sect. 4.5), pixels from frames of differing exposure length are rescaled and averaged together. The dynamic range is determined by the readout noise of the longest 
exposure which is not rescaled, and thus for the USI with $c_{\text {sat }}=65535$ this gives $84 \mathrm{~dB}$. This is somewhat misleading, however, because rescaling the shorter exposures decreases signal-to-noise ratio by up to a factor of 4 (when the only the shortest exposure is used). Therefore, while the improved dynamic range from HDR imaging can better capture the wide intensity range of the sky, it comes at a cost of increased noise for darker pixels.

The use of an interline transfer CCD is not without tradeoffs. Smear is very apparent in images with direct sun exposure. Smear has two sources: (1) stray light entering the VCCD (vertical transfer CCD) during readout; (2) charge generation occurring deeper in the silicon photodiode layer that diffuses to any of the charge collection or transfer electronics. The VCCD is the interline column near the exposed photodiode column, and is where the vertical readout step is performed. Longer wavelengths penetrate further into the silicon before being absorbed and can generate hole-electron pairs in undesirable locations. This is why the smear is noticeably worse in the red channel of Fig. 3b. Blooming, which is apparent as a saturated border of bright objects, is another problem for CCDs, and is noticeable in USI imagery near the sun. It is not serious however, because each KAI-04022 pixel has a vertical overflow drain to prevent large amounts of charge from diffusing to nearby collection sites.

\subsection{Enclosure and balance of system design}

For solar forecasting, tough environmental conditions such as hot and dusty deserts will be encountered. The USI is designed to survive $60^{\circ} \mathrm{C}$ ambient air temperature and direct sunlight conditions. It has a light-colored exterior to reduce shortwave absorption and has two $80 \mathrm{~W}$ thermoelectric coolers with a NEMA $4 \mathrm{X}$ rating. To monitor the system's environmental health, a suite of temperature and relative humidity sensors was added to measure camera, power supply, internal and external ambient, and dome conditions. The internal enclosure walls are all insulated to reduce thermal conductivity of the enclosure, which with the use of active thermal control, keeps it cooler on hot days and warmer on cold days. Internal water condensation was initially found to be an issue. Improved system sealing and thorough water testing was found to be necessary. Three $20 \mathrm{~W}$ resistive heating strips were installed on the base of the dome to reduce condensation on the exterior dome surface.

The USI camera is controlled by a $1.8 \mathrm{Ghz}$ dual core (Atom D525) embedded computer running Linux Ubuntu 12.04. The images can be stored locally on a set of internal and USB hard drives, or it can be transferred across a network connection. Using an embedded computer gives the system flexibility for customizing the configuration on a per deployment basis, and the capture software can easily be reconfigured, reprogrammed, or debugged remotely. A labeled CAD model of the USI is shown in Fig. 1.

\section{System operation}

\subsection{Image capture and storage}

Images are received from the camera as uncompressed single-channel 12-bit images with per-pixel color determined by the CFA. After three exposures are composited in the HDR process (Sect. 4.5), the combined image is still a single channel, but with 16-bits per pixel. Images are compressed and stored in a lossless 16-bit PNG format as a single channel image. A single pixel contains information about only one color of red, green or blue light. To produce a full color image from the pixel array suitable for processing, linear demosaicing is applied prior to use. Current image sizes are around $3 \mathrm{MB}$ per image, which when capturing images every $30 \mathrm{~s}$ during daylight hours requires between 3 and $6 \mathrm{~GB}$ day $^{-1}$ depending on the time of year.

The maximum frame rate of the USI system in single exposure mode is $15 \mathrm{fps}$, which is relatively low. Future dynamic computer vision approaches to solar forecasting (e.g., optical flow) may require higher frame rates, and for these methods, the camera used on the USI may not be suitable. In $H D R$ mode, which is the standard USI operational mode, three images are captured sequentially in $160 \mathrm{~ms}$, which is a frame rate of $18.8 \mathrm{fps}$ (or HDR frame rate of $6.3 \mathrm{fps}$ ). This increase in frame rate is possible because a smaller $1748 \times 1748$ region of interest, extracted from the center of the $2048 \times 2048$ pixel array, is transferred off the camera. After subsequent HDR compositing and PNG image compression, the effective frame rate drops to $0.77 \mathrm{fps}$ (i.e., $1.3 \mathrm{~s}$ per HDR image).

\subsection{System monitoring and control}

The raw images generated by the camera are inconvenient for qualitative inspection on a user's screen because they are not in color (raw Bayer format), the file sizes are relatively large so loading is slow, and a majority of the sky resides within the lower end of the 16-bit dynamic range which means the image appears very dark except for the sun. Preview images are therefore generated, which are full color, but lower resolution, compressed, and tone-mapped to 8 bits per color channel. These previews are small enough to be uploaded to the operator from all sites - including remote ones using a cellular modem - and allow the image quality and availability to be inspected at a glance.

In addition, the data acquisition system reports temperature and humidity every $30 \mathrm{~s}$. The internal temperature and dome temperature are used to control the heaters and coolers in the USI to ensure that the critical electronics are always within their operating temperature bounds, and to avoid conditions that might lead to condensation. A live plot of temperature and humidity is uploaded to the operator. An important feature of the microprocessor controlled data acquisition system is its ability to automatically power-cycle the USI computer if it fails to respond. This has proven to be a valuable 
backup, particularly on remote systems that are hard to access and crash more often than the others due to bugs in the cellular modem driver.

\section{Imaging performance characterization}

\subsection{Noise sources and pixel photoresponse}

Each pixel in a camera is an independent radiometric sensor, and has small response variations from its neighbors due to small manufacturing differences. After charge is collected on a pixel, it is converted to a voltage and then to a digital value, and at each step in the process noise is introduced. Common sources of noise include dark current generated by the semiconductor in the bulk and at the surface, reset noise from charge to voltage conversion (which is typically minimized by correlated double sampling), read noise from the camera's readout electronics, and photoresponse nonuniformity (PRNU) arising from small manufacturing differences of each pixel. Because there is a consistent spatial variation of many of these noise sources, it often forms a pattern called fixed pattern noise (FPN). Shot noise, arising from the quantum nature of the photons generating the signal, occurs in all imaging systems and acts as a lower bound to measurement uncertainty. It adds a random element to each image that is Poissonian in nature and it can only be reduced by averaging frames, which is not feasible for fast-moving clouds or when high frame rates are desired.

Each pixel's response can be characterized and corrected so that under the same illumination, the corrected output is the same when averaged over several frames. A comparison of the average of several frames is required because shot noise will always be present in an individual frame. A polynomial can be used to model a pixel's response to light:

$c_{i j}(I, t)=\sum_{n=0}^{N} \hat{a}_{i j, n} t^{n}+\sum_{m=1}^{M} d_{i j, m}(I t)^{m}$,

where $c_{i j}(I, t)$ is the camera measurement in counts at the $i$, $j$ pixel location, $I$ is the irradiance incident on the pixel, $t$ is the integration time of the exposure, $\hat{a}_{i j, n}$ are coefficients that characterize the individual pixels' dark response, and $d_{i j, m}$ characterize the pixels' photoresponse. Sensor noise and response characteristics are temperature dependent, so coefficients $\hat{a}_{i j, n}$, and $d_{i j, m}$ will also vary with temperature. Here it has been assumed that dark response and photoresponse can be separated.

To determine the coefficients in Eq. (1), the irradiance $I$ on the sensor plane must be known, which when using a lens implies the scene radiance must be known over the entire field of view. This can be achieved with a calibrated flat-field source. Many of the components of solar forecasting algorithms (e.g., Chow et al., 2011; Yang et al., 2014) have a training step where either relative brightness or brightness ratios are used to determine thresholds, or texture information is used and therefore calibrated radiance is not needed. Instead, these algorithms require spatially consistent measurements (i.e., consistent between pixels), for which a simpler radiometric uniformity correction (Sect. 4.3) can be used. This has the advantage that it can also be employed in the field after the instrument has been deployed. The camera output signal $s_{i j}$ after radiometric uniformity correction can be written as:

$s_{i j}(I, t)=\sum_{m=0}^{M} b_{i j, m}\left(c_{i j}(I, t)-a_{i j}(t)\right)^{m}$,

where $a_{i j}(t)$ provides the dark-field correction, and coefficients $b_{i j, m}$ provide the flat-field (i.e., uniform illumination) correction.

The parameters of the radiometric uniformity correction $a_{i j}$ and $b_{i j, m}$ have temperature dependencies that are not treated in the formulation of Eq. (2) or developments to follow. Sky imaging systems expecting large changes in sensor and camera temperature should perform the testing described in Sects. 4.2-4.4 at different temperatures to better understand the impacts. For the USI, the dark current of KAI04022 roughly doubles for every $9^{\circ} \mathrm{C}$ increase in temperature in the system operating range. The USI camera temperature, measured with an LM335 thermal probe attached to the camera body, has been observed to change by over $20^{\circ} \mathrm{C}$ between day and night.

\subsection{Dark response}

The dark response of the sensor was measured by recording images in complete dark (unlit room, USI enclosure closed, lens cap on and covered with a thick, opaque cloth) at several integration times. Raw 12-bit images were taken at 25 different integration times ranging from $1 \mathrm{~ms}$ to $2 \mathrm{~s}$, and the sequence was repeated ten times for a total of 250 images. If the thermally generated dark current is low in comparison with the bias (defined below), there should be little increase in measured dark response signal as a function of integration time, i.e., $a_{i j}(t)$ should not vary with time. The low dark current of the USI is illustrated in Fig. $4 \mathrm{a}$ as a set of probability density functions (PDFs) showing the occurrence frequency of each measured dark response count value. The average of ten frames at each integration time is used to reduce random noise present in a single measurement. PDFs of the difference between a $1 \mathrm{~ms}$ average image frame (averaged from nine, $1 \mathrm{~ms}$ exposures) and a single frame at each exposure time are shown in Fig. 4b. Both sets of PDFs show no strong change as a function of exposure time which confirms the thermally generated dark current for the USI is low. The A/D converters that convert voltage of each pixel to digital counts are calibrated to provide on-scale measurements throughout the range of the sensor. This sets the lower dark bound (or bias) to always be above zero, which for the USI camera this centered at approximately 40 counts (or $\sim 1 \%$ of full scale, see dark bias distribution Fig. 4a). 

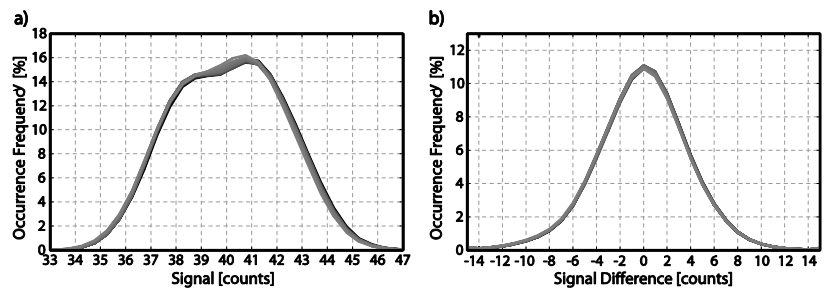

Figure 4. (a) Occurrence frequency of signal measured in a dark room for 25 different integration times, ranging from $1 \mathrm{~ms}$ (black) to $2 \mathrm{~s}$ (lightest gray). Ten exposures at each integration time were averaged to construct each PDF. (b) Occurrence frequency of the signal in a single frame for the 25 integration times with an average $1 \mathrm{~ms}$ frame subtracted. Individual labels for each integration time were not added because curves are not discernable.

The temporal component of the dark response for the exposure times used on the USI $(<1 \mathrm{~s})$ is small, but there is still a spatial component of the dark response called fixed pattern noise (FPN). The FPN is shown in Fig. 5a. There is relatively little variation within each column. Two distinct image halves are noticeable, an artifact caused by the use of two $\mathrm{A} / \mathrm{D}$ converters, each serving half the sensor. Columns near the center of each half have lower readouts than columns near the edges. The dark FPN can be removed by subtracting the measured dark response to obtain the dark field corrected signal $s_{i j}^{d}(I, t)$

$s_{i j}^{d}(I, t)=c_{i j}(I, t)-a_{i j}(t)$,

which is the same as the term in parenthesis in Eq. (1). The dark image term $a_{i j}(t)$ is obtained by averaging several frames at integration time $t$. An image appears much more uniform after dark correction (Fig. 5b) which indicates the FPN has been eliminated. For over $99.9 \%$ of pixels, $a_{i j}(t)$ does not show significant variation with time, however a small number of "hot" pixels have higher than average dark current and/or a nonlinear temporal dark response, and thus the time dependence of $a_{i j}$ is retained.

\subsection{Sensor photoresponse uniformity correction}

Photoresponse nonuniformity is caused by differing gains on each photodetector in the focal plane array; i.e., $d_{i j, m}$ in Eq. (2) differs slightly for each pixel. The most direct approach to PRNU correction uses flat-field measurements (uniform lighting over the entire field of view) in order to adjust each pixel so that its response is uniform under uniform illumination. An alternative method is to use an illumination source that produces a smooth image without large brightness gradients. The resulting image can then be fit with a surface, and deviations of a given pixel from this surface can be considered the non-uniformity of that pixel. At each integration time, 10 exposures are used to obtain an average of the dark corrected signal $s_{i j}^{d}(I, t)$ so that the effects of shot noise
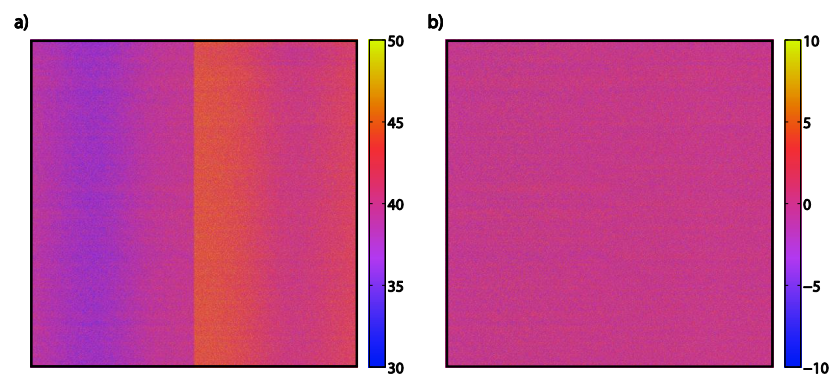

Figure 5. (a) An example dark frame for a $100 \mathrm{~ms}$ exposure and (b) the corrected dark frame. Typical pixel values in (a) range from 32 to 47 with a mean around 40 counts (of $2^{12}$ ).

are reduced (the 10-frame average denoted by $\overline{s_{i j}^{d}(I, t)}$ ). The same integration times used for the characterizing the dark response in Sect. 4.2 are used. At each integration time, a 5 th order surface (denoted $\left\langle s_{i j}^{d}(I, t)\right\rangle$ ) is then fit to the average dark corrected signal $\overline{s_{i j}^{d}(I, t)}$ as a function of pixel location $(i$ and $j)$. The resulting set of surfaces $\left\langle s_{i j}^{d}(I, t)\right\rangle$ is used to determine the coefficients $b_{i j, m}$ as a function of exposure time $t$ :

$\left\langle s i j^{d}(I, t)\right\rangle=\sum_{m=0}^{M} b_{i j, m}\left(\overline{s_{i j}^{d}(I, t)}\right)^{m}$,

where for each pixel $i j$, both $\overline{s_{i j}^{d}(I, t)}$ and $\left\langle s i j^{d}(I, t)\right\rangle$ are a function of position $(i, j)$ and exposure time (here we assume the scene brightness is not changing, thus $I$ is constant). The surface fit also assumes that if a CFA sensor is used, separate surface fits are used for each color channel. Before fitting a surface to $\overline{s_{i j}^{d}(I, t)}$ (Fig. 6c) at each integration time, a rowby-row adjustment was applied to remove the imbalance in output from the A/D converters. A low-order fit of the rowby-row ratio of two columns on either side of the border between image halves was used to adjust the left side of the image.

An example of the results of the uniformity correction for the red channel is shown in Fig. 6. For this figure, the terms $b_{i j, m}$ in Eq. (4) are obtained by using a training set of images, setting $M=2$. The correction is then applied to a validation set using Eq. (2). The method corrects hot pixels that have not reached saturation, and corrects small-scale FPN, but it fails to correct large-scale nonuniformity. This occurs because the surface used for correction is fit to non-uniformities that occur across the whole image. It is therefore not as robust as the uniform illumination approach but is a useful substitute in field operations. This approach to uniformity correction can also be used in the field to help identify and quantify localized sensor degradation due to direct sun exposure. 


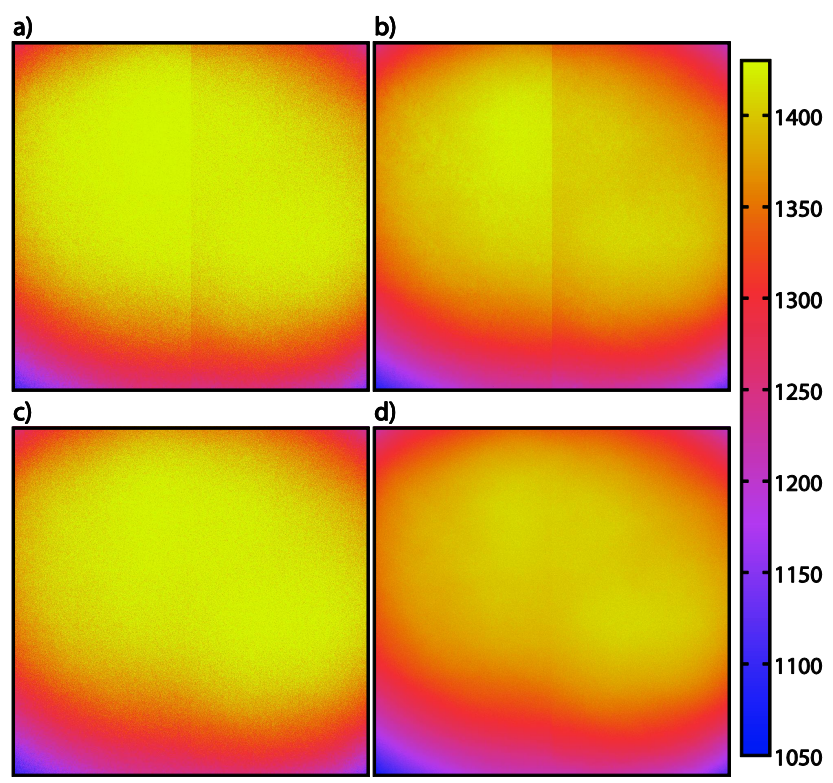

Figure 6. (a) Raw red image of smooth light source obtained by sub-sampling only red pixels from the color filter array; (b) average of ten red frames, including (a); (c, d) uniformity correction applied to $(\mathbf{a}, \mathbf{b})$, respectively.

\subsection{Photoresponse linearity}

Knowledge of the camera's response as a function of both intensity and exposure time is a prerequisite for the HDR process. The simplest model for a pixel's photoresponse is linear in the product of irradiance $I$ on the sensor plane and exposure time $t$

$c_{i j}(I, t)=\hat{a}_{i j, o}+d_{i j, 1} I t$,

where $M$ and $N$ from Eq. (1) have been taken as zero and one, respectively. Assuming a constant irradiance during the exposure sequence, we convert the value measured in an exposure of integration time $t$ to the expected value had it been captured at integration time $t_{\text {ref: }}$ :

$c_{i j}\left(I, t_{\mathrm{ref}}\right)=\left(c_{i j}(I, t)-\hat{a}_{i j, o}\right) \frac{t_{\mathrm{ref}}}{t}+\hat{a}_{i j, o}$.

This linear model predicts that the measurement values of the same scene should be scaled by the ratio of the exposure times from one image to the next. For example, we would expect that all the values in a $6 \mathrm{~ms}$ exposure would be 4 times as large as the values of the corresponding pixels in a $1.5 \mathrm{~ms}$ exposure. Figure 7 shows the ratio of modeled values based on a longer exposure to the measured values in a shorter exposure (i.e $t_{\text {ref }} / t=0.25$ ). An average of five frames was used at each exposure time in making the comparison. To avoid negatively biasing the results, pixels that saturate in the longer image were removed, which corresponds to pixel values of over 1024 in the shorter exposure.
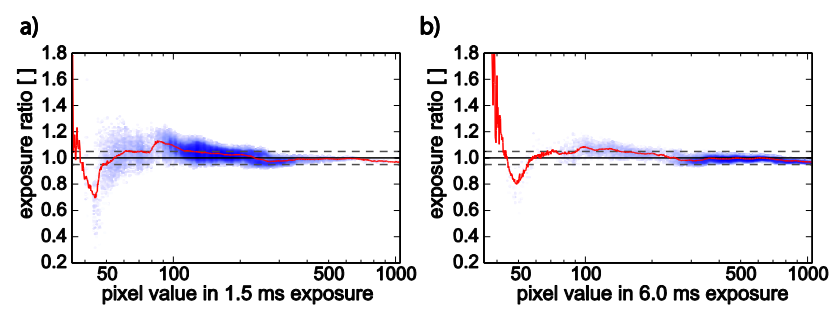

c)

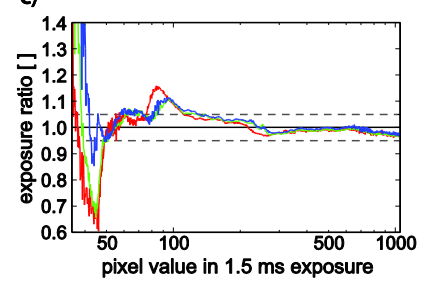

Figure 7. Evaluation of sensor linearity using sky images under thin overcast conditions. In (a), a point cloud (and median in red) showing the distribution of the ratio between a $6 \mathrm{~ms}$ exposure and a modeled $6 \mathrm{~ms}$ exposure generated from a $1.5 \mathrm{~ms}$ exposure, as a function of measured value in the $1.5 \mathrm{~ms}$ image. In (b), the same as (a), but with 6 and $24 \mathrm{~ms}$ exposures. In (c) the median line for each color is shown. To reduce random noise, each of the compared images is the average of five exposures captured over the course of approximately $3 \mathrm{~s}$.

The observed deviation from unity is a measure of the error we introduce by scaling up a given value from the short exposure to place it in a composite with the longer exposure. Below 100 counts $(\sim 2.5 \%$ full scale), there appear to be significant non-linearity effects, and we do not recommend using signals below this level. Between about 400 and 800 counts, the median deviation is nearly zero. Deviations are small $(<5 \%)$ from around 150 counts to the end of the overlap range just above 1000 counts. Over the majority of the range, neither exposure time nor color has a significant effect on the result. The overlap of this "sufficiently linear" region on the abscissa of Fig. 7 extends from 409 counts (the lower limit in the short exposure) to 921 counts (the upper limit in the long exposure after multiplying by the integration time ratio, i.e., $3684 \times 0.25=921$ ). We have therefore elected, for the purposes of this work, to consider pixel response to be sufficiently linear if the value is between 10 and $90 \%$ of full scale, i.e., 409 to 3686 counts.

\subsection{High dynamic range imaging}

In order to image the daytime sky it is important that the camera have a large dynamic range, since we wish to obtain images of both very bright objects (such as the sun and sunlit clouds) as well as very dark objects, such as the undersides of thick clouds. Unfortunately, 12 bit (or fewer) image sensors generally do not have sufficient dynamic range for this task in a single exposure. Instead, we capture multiple exposures with different integration times in quick succession and com- 


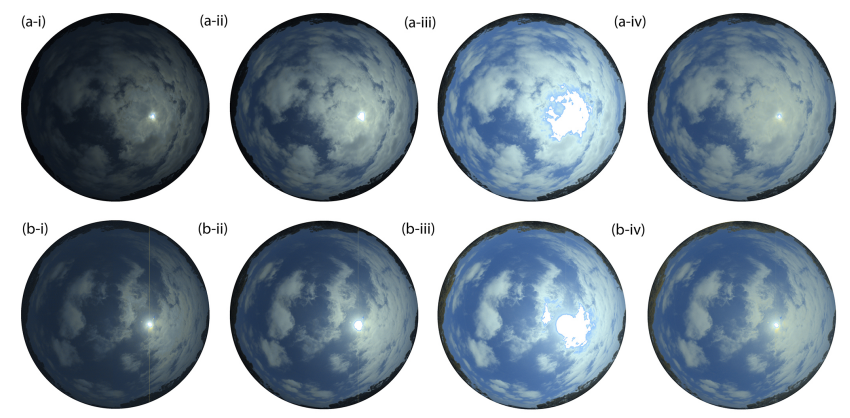

Figure 8. USI 1.2 high dynamic range (HDR) exposure sequence for (a) 23 May 2013, 03:22 pm PDT and (b) 23 May 2013, 02:48 pm for integration times of (i) 30, (ii) 120, and (iii) $480 \mathrm{~ms}$. (a-iv) and (b-iv) show the final HDR composites.

bine those exposures into a single high dynamic range image (Debevec and Malik, 1997). Three 12-bit exposures are composited together to produce a single 16-bit image.

Although methods exist that would allow us to use a more sophisticated photoresponse model than Eq. (5) (e.g., Mann and Picard, 1994), by only using pixels in the linear region of the sensor photoresponse (Sect. 4.4), we can apply the simple linear response model without significant error. For purposes of the HDR composite, this means that for a single exposure the pixels with values below 409 or above 3686 counts are excluded. The integration times on the USI are separated by factors of four (i.e., $t, 4 t$, and $16 t$, where $t$ is system dependent). This ensures that the region between 409 and 921.5 counts in a shorter exposure will overlap with the region between 1636 and 3686 counts in a longer exposure. Based on the results shown in Fig. 7, these settings ensure the linear approximation in Eq. (5) is applicable for the subset of overlapping pixels in the HDR image.

The HDR process is straightforward. First we select the pixels in each of the three exposures that are properly exposed, eliminating areas that are below 10 or above $90 \%$ of full scale. Next, using Eq. (6), we map the values for each pixel to what they would have been in the frame with the longest exposure time. This assumes that for the short duration of an HDR exposure sequence, scene intensity is constant. Finally, we combine the exposures, using the average of all valid values for each pixel. This method is simple and effective, as demonstrated in Figs. 8 and 9. It is, however subject to small composition artifacts if the sensor response linearity is not properly characterized. If an image patch contains values for which sensor response is nonlinear and the HDR algorithm transitions from using a different subset of the three available exposures within this patch, a small 12 pixel intensity step will occur which, after demosaicing into a color image, appears as a color fringe.

Figures 8 and 9 demonstrate the HDR method applied to two systems, USI 1.2 and USI 1.8 respectively (see Table 3 ). USI 1.2 used a $9520 \mu \mathrm{m}$ diameter aperture and neutral density

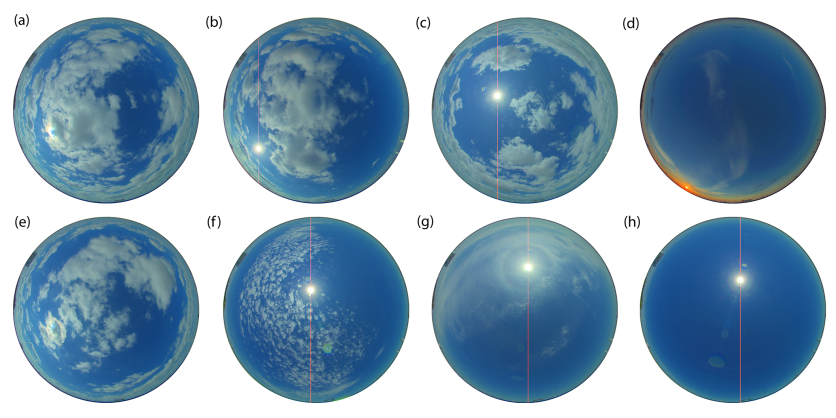

Figure 9. HDR images from USI 1.8 in April and May 2013, showing a variety of sky conditions. Images required intensity rescaling for display purposes.

filter, whereas USI 1.8 used a modified aperture of diameter $1000 \mu \mathrm{m}$ (note the spectral variation between instruments). Figure 8a and $\mathrm{b}$ highlights the differences between the HDR capture sequence in cloudy conditions for an obstructed and unobstructed sun. Figure 9 provides an overview of imaging performance in a variety of sky conditions, with both obstructed and unobstructed sun. Figure $9 \mathrm{~d}$ shows a thin cloud in low lighting conditions, and in Fig. 9g a halo caused by the thin clouds can be seen.

\subsection{Brightness measurement uncertainty in HDR imagery}

Two images of the exact same scene will not be identical due to the random shot noise present in the measurements. Electron generation in the sensor follows a Poisson distribution, so the root mean square (rms) of the shot noise is expected to be $e_{i j \text {,shot }}=\sqrt{e_{i j}}$, where $e_{i j}$ is the quantum unit being measured at pixel $i, j$. The quanta considered here is electrons. Assuming shot noise is the dominant noise source, this square root increase in rms shot noise with stored electric charge $e_{i j}$ implies the signal-to-noise ratio also increases as $\sqrt{e_{i j}}$. Shot noise places a fundamental limit on the lower bound of measurement uncertainty for an image sensor. The predicted rms noise as a function of count value for a 12-bit image is shown in Fig. 10a. For this calculation, the manufacturer specified gain $g$ of 0.174 counts per electron was used. Measured system noise as a function of pixel value (in counts) was quantified by computing the pixel-by-pixel standard deviation $\sigma_{i j}$ for ten frames of a stationary scene, binning $\sigma_{i j}$ by the pixel-by-pixel mean $\mu_{i j}$ into bins $0-4095$, and finally by taking the median $\sigma$ of each bin. The dropoff near the maximum occurs because the upper bound that the saturation limit imposes causes the standard deviation of measured values to reduce.

When combining exposures in an HDR composite, the shot noise present in an individual pixel will depend on which exposures were compiled for that particular pixel, and the scaling factor $t_{\text {ref }} / t$ for each pixel in the composition. For a sufficiently large number of electrons, the Poisson distribu- 
Table 3. USI Locations in the United States and deployment time ranges.

\begin{tabular}{lrrrllll}
\hline USI no. & Longitude $\left({ }^{\circ}\right)$ & Latitude $\left({ }^{\circ}\right)$ & Altitude $(\mathrm{m})$ & State & City & Start date & Stop date \\
\hline 1.1 & -117.233088 & 32.881090 & 120 & California & La Jolla & 21 Apr 2012 & - \\
1.2 & -117.240987 & 32.872136 & 135 & California & La Jolla & 6 Jun 2012 & - \\
1.5 & -117.243111 & 34.076355 & 347 & California & Redlands & 18 Oct 2012 & Mar 2014 \\
1.6 & -117.209333 & 34.079822 & 384 & California & Redlands & 23 Oct 2012 & Mar 2014 \\
1.7 & -97.478766 & 36.618377 & 304 & Oklahoma & Billings & 11 Mar 2013 & 4 Nov 2013 \\
1.8 & -97.484871 & 36.604094 & 318 & Oklahoma & Billings & 11 Mar 2013 & 4 Nov 2013 \\
1.9 & -117.238378 & 32.707122 & 15 & California & San Diego & 19 Apr 2013 & Mar 2014 \\
1.10 & -156.479136 & 20.890549 & 20 & Hawaii & Kahului & 21 Aug 2013 & - \\
\hline
\end{tabular}
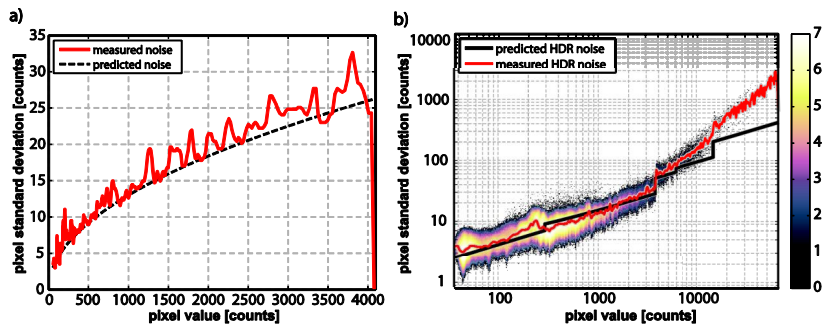

Figure 10. Photon transfer curve for a USI system for (a) a 12bit image, and (b) an HDR image. The theoretical minimum shot noise limit is shown as a black line, and the median of the noise distribution at each count value is shown in red. In (b), the density of the pixel standard deviation distribution is shown behind the curves.

tion is approximately normal by the central limit theorem, and thus the rms noise (which is the same as the standard deviation of the noise since the mean is zero) from each frame can be summed in quadrature to obtain the rms shot noise $c_{i j, \text { shot }}$ in an HDR exposure, i.e.,

$c_{i j, \mathrm{shot}}=g \sqrt{\sum_{k}^{P} \frac{t_{\mathrm{ref}}}{t_{k}}\left(e_{i j, \mathrm{shot}}^{2}\right)_{k}}$,

where $k$ is the individual frame index, $P$ is the number of frames, which ranges from one to three in this work. The actual rms noise present in an HDR image was computed using the method described for Fig. 10a, and is shown in Fig. 10b. The noise is compared to the shot noise limit (Eq. 7, black line, Fig. 10b), where the number of frames in the HDR composition is determined using the algorithm described in the previous section. The use of different combinations of frames can be seen as sharp jumps in the theoretical minimum in Fig. 10b.

The curves presented in Fig. 10 are similar to photon transfer curves (PTCs) which characterize not only shot noise, but all random noise present in the image sensor. Noise sources such as dark current and read noise are subtracted out of a PTC. The closeness of the curves to the shot noise limit indicates that for the USI system, sources of noise other than shot noise are small in both the 12-bit image, and the HDR composition. The fluctuations in each curve, and the dips below the theoretical minimum occur because a limited number of samples were taken (10 frames). Above 15000 counts, very few samples were present in the HDR images, so noise in this region is not well characterized here.

\subsection{Stray light}

The red-blue-ratio image ( $R B R)$, defined as the ratio of the red channel to the blue channel, is the most common feature used for cloud detection. Clear sky has a relatively low RBR and clouds have a higher RBR. RBRs typically span between 0.4 and 1.2 for the USI, and the threshold for cloud is about 0.5 . Stray light, due to exposure of the optical assembly to the direct beam, results in spots and artifacts in the image that are brighter and more spectrally neutral than they should be, resulting in either false positive cloud detections when the stray light pushes a hazy sky above the cloud threshold, or missing clouds due to contamination of the clear sky library (see Chow et al., 2011, or Yang et al., 2014, for details).

In order to characterize the stray light present in our system, we used a simple, hand-held shade to block the sunlight. The shade used was not much larger than the dome and was held at a considerable distance so as to sufficiently shade the entire optical assembly while minimizing the number of pixels occupied by the shade within the image. Measurements were conducted on a clear day (13 May 2013) and shaded and un-shaded images were taken $30 \mathrm{~s}$ apart. By comparing images captured with and without the shading device, we can observe the effect of stray light on the resulting images. Three different pairs of images are compared in Fig. 11. First, a normal image is compared to one taken with the dome removed. Second, with the dome removed, images taken with and without the shade are compared. The third and final comparison considers shaded and un-shaded images with the dome on. The latter comparison gives the best estimate of the total effect of stray light on the images produced by the USI, while the first two allow us to qualitatively separate effects due to the dome and the lens. To quantify the effects of stray light, the residual fractional intensity $\left(I_{2}-I_{1}\right) / I_{1}$ is computed and shown in the left column of Fig. 11, where $I_{1}$ is the image with the shade (or without dome, pair a), and $I_{2}$ is the image without the shade (or with dome, pair a). 

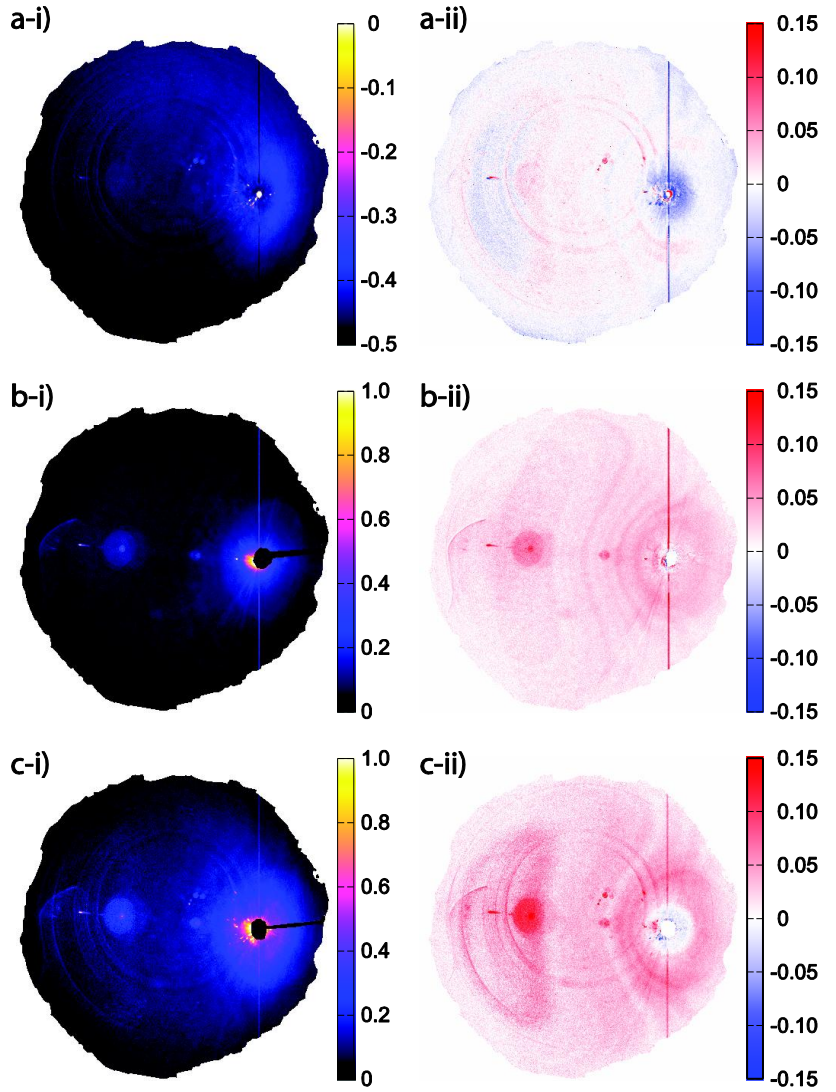

Figure 11. Stray light from the dome (top), lens and neutral density filter (middle), and whole system (bottom). The left column shows the fractional change in intensity due to stray light, while the right column shows the shift in the red-blue ratio from the shaded to unshaded image. Images were recorded against a clear (blue) sky, so stray light shifts toward the red. Note the scale change between (a) and $(\mathbf{b}, \mathbf{c})$ in the left column.

The following stray light effects were identified: (1) an overall increase in measured intensity averaging $12 \%$ across the image (Fig. 11c-i); (2) concentric ring-like reflections off the front face of the camera lens that reflect off the innersurface of the dome (Fig. 11a vs. b); (3) particularly strong (and bluish) forward scattering off the dome (bright circle in Fig. 11a-ii); (4) sharp reflections off of elements in the optical assembly, visible as spots along the intersection of the solar principal plane and the image plane (all); (5) a "swoopy" shape resulting from reflection of sunlight off the rear gelatin neutral density (ND) filter at the back of the lens (all); and (6) vertical smear that results near the sun from signal overflow during sensor readout (all); (7) at higher solar elevations (Fig. 12), a reflection of the sun off the surface of the image sensor. Here, the solar principal plane is defined by camera optical axis and the vector to the sun. The dome decreases the average image intensity by about $46 \%$ because of the ND acrylic used (Fig. 11a vs. b and c). While the dome surface was clean during testing, in normal operations dirt

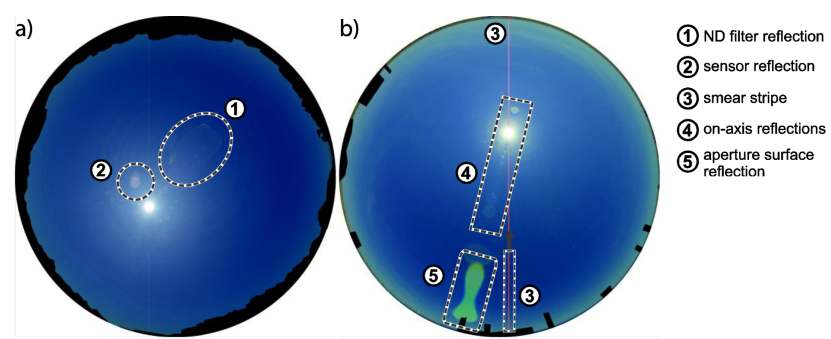

Figure 12. Stray light comparison between two designs of the USI; (a) design with filter, and (b) design with modified aperture.

or scratches on the dome will result in additional scattering with a specific pattern that changes not just with the position of the sun, but also as a function of time since last cleaning.

Stray light impacts of the modified aperture (Sect. 2.1) versus the ND filter were qualitatively evaluated by visually inspecting clear sky images such as those in Fig. 12. The following differences between the modified aperture and the wide-open, filtered configuration are noted: (i) reflection from the ND filter surface is, naturally, missing in the model without a filter; (ii) the $9250 \mu \mathrm{m}$ aperture in the filtered configuration exhibits a pair of reflections of the sun striking the image sensor that become visible at high solar elevations (when the direct-beam is nearly orthogonal to the image plane); this has not been observed using the modified aperture; (iii) the modified aperture shows a larger number of circles along the diameter containing the sun (i.e., intersection of the solar principal plane and the image plane); (iv) a "feathery" radial pattern is sometimes observed near the sun with the modified aperture, arising from imperfections in the circularity of the aperture; (v) the modified aperture has a more prominent smear stripe because the selected aperture diameter allows more light into the camera; and (vi) prototypes with extremely small apertures exhibited diffraction rings around the sun (Fig. 3). Effect (iii) occurred because the antireflective black-oxide coating applied to the steel was mistakenly polished by the machinist, which increased its reflectivity.

Increases or decreases in residual fractional intensity affect the radiometric analysis of sky imagery, but for solar forecasting primarily the RBR is of interest. Therefore, it is primarily spectral variations in stray light that are of interest. Stray light is expected to increase RBR because a majority of stray light originates from the direct solar beam, which is whiter than most of the sky. To quantify the impacts of stray light on cloud detection, the difference RBR2 - RBR1 of each of the three described pairs is shown in Fig. 11-ii. Figure 11a-ii indicates that the dome decreases RBR in the region directly around the sun, and in the region $90^{\circ}$ from the sun along the solar principal plane. When the effects of the dome are compared to a shaded image (Fig. 11c-ii), however, the RBR increases everywhere except in the immediate vicinity of the sun, with the lens-face reflections (concen- 
tric circles) and the region $90^{\circ}$ from the sun along the solar principal plane being the most prominent features. The clear sky library (CSL, Chow et al., 2011) is built from un-shaded images with the dome on (e.g., the RBR images used to construct Fig. 11c-ii), and stray light features are included in the cloud detection thresholds. Many of the stray light features are captured well by the CSL because they are functions of both solar zenith and sun-pixel angle. This becomes problematic when the sun is shaded by clouds because the features are not present. This leads to significant problems detecting cloud near the sun, because as clouds pass and intermittently shade the sun, the RBR of clear sky and the clouds fluctuates, and a single threshold becomes problematic (Yang et al., 2014).

To reduce the impact of stray light, we have performed experimentation with a stray light ratio lookup table as a function of solar zenith angle, sun-pixel angle, and image zenith angle (similar to the clear sky library, Chow et al., 2011). However, the results, while promising, were inconsistent and thus are not reported here. A more robust approach based on generating synthetic, stray-light-free images with a 3-D radiative transfer model is currently being investigated. From our experience using the USI for forecasting, the stray light features discussed here negatively affect image quality and result in identifiable forecast performance degradation. Yang et al. (2014) have implemented adjustments to the cloud detection methods of Chow et al. (2011) to specifically address solar power forecast errors due to stray light. In future work we hope to develop corrections for the USI imagery so that stray light levels in imagery are reduced prior to being input into the cloud detection algorithms.

\subsection{Color balancing}

The neutral density filters currently used in the USI (Kodak Wratten 2, no. 96 ND3.0) introduce a color cast to the image. Basic color correction is performed by selecting a region of cloud that should be a neutral grey color and scaling the red, green, and blue signals relative to each other such that neutral grey is achieved. This color correction has been applied to many of the images shown above, and is useful when converting RGB images to other color spaces such as HSV, but has little effect on the red-blue-ratio. In the future we may use a color reference chart (e.g., the IT8.7/2-1993 calibration target) in order to improve the color balance of USI images in a way that might impact forecasting performance more.

\section{Deployment experience}

The UCSD USI system has been deployed across the United States (Table 3). The predominant cloud types in coastal California (USIs 1.1, 1.2, 1.9) are marine stratocumulus. In Kahului, Hawaii there are persistent orographic clouds over the West Maui Mountains to the west-northwest of USI 1.10 which makes it an interesting place to study non-advective solar forecast schemes. Redlands, California is hot and dry, and usually clear, but often sees higher ice clouds and larger synoptic systems. In Billings, Oklahoma there is a wide diversity of cloud conditions that occur from high ice clouds, to lower cumulus clouds. Solar forecasting algorithms may have location dependent performance, and testing components of an algorithm in multiple locations can help to identify shortcomings and areas for improvement.

The data gathered from the two instruments in Billings, Oklahoma are of particular interest because they were fielded at a United States Department of Energy Atmospheric Radiation Measurement Program field site (the Southern Great Plains site). The site includes a diverse suite of measurement equipment, including cloud radar covering a number of bands, several lidar systems, shortwave and longwave radiometers, aerosol measurements, and a Doppler wind profiler. These collocated measurements will be used to assess the performance of a number of remote sensing algorithms developed for the USI.

\section{Conclusions and future work}

Clouds have a high degree of spatial complexity and the intensity range within a single scene can be over five orders of magnitude (including the sun). For solar forecasting applications, it is important to capture this information at a high spatial and radiometric resolution to facilitate the development of advanced algorithms and techniques. The UCSD Sky Imager system is a step in this direction. Ten instruments have been built and can be made available to other researchers. The units come with a camera and system control software and an extensive library of processing tools is available. The developers are also open to commercializing the instrument and extensive design documentation is available.

\begin{abstract}
Acknowledgements. This work would not have been possible without extensive student support in the design, fabrication, and assembly of the USI. We would like to thank, in no particular order, Caspar Hanselaar, Edmundo Godinez, William Gui, Felipe Mejia, Prithvi Sundar, Dan Erez, Scott Kato, Amy Chiang, Jessica Traynor, Kristen Ostosh, Tyler Capps, Sebastian Schwarzfischer, Sebastian Pangratz, Christian Faltermeier, Nick Truong, Salil Kektar, Jeff Yeh, Max Twogood, Alex Turchik, Danielle Donnely, Emily Davis, and last, but not least the Victors Fung (1) and Piovano (2). We also appreciate funding from the Panasonic Corporation, the Department of Energy High Solar PV Penetration Award Number EE-0004680, and the California Energy Commission contract 500-10-060.
\end{abstract}

Edited by: M. Hamilton 


\section{References}

Anderson, M.: Studies of the woodland climate: I. The photographic computation of light conditions, J. Ecol., 52, 27-41, doi:10.2307/2257780, 1964.

Bayer, B. E.: Color Filter Array, U.S. Patent No. 3,971,065, U.S. Patent and Trademark Office, Washington, DC, 1975.

Brown, H. E.: The canopy camera, Station Paper 72, Fort Collins, CO: U.S. Department of Agriculture, Forest Service, Rocky Mountain Forest and Range Experiment Station, 1962.

Cazorla, A., Olmo, F. J., and Alados-Arboledas, L.: Development of a sky imager for cloud cover assessment, J. Opt. Soc. Am., 25, 29-39, doi:10.1364/JOSAA.25.000029, 2008.

Chow, C. W., Urquhart, B., Lave, M., Dominguez, A., Kleissl, J., Shields, J. E., and Washom, B.: Intra-hour forecasting with a total sky imager at the UC San Diego solar energy testbed, J. Sol. Energ., 85, 2881-2893, doi:10.1016/j.solener.2011.08.025, 2011.

Debevec, P. and Malik, J.: Recovering high dynamic range radiance maps from photographs, Proceedings of the ACM SIGGRAPH'97, Los Angeles CA, 369-378, 1997.

Dupree, W., Morse, D., Chan, M., Tao, X., Iskenderian, H., Reiche, C., Wolfson, M., Pinto, J., Williams, J. K., Albo, D., Dettling, S., Steiner, M., Benjamin, S., and Weygandt, S.: The 2008 CoSPA forecast demonstration (collaborative storm prediction for aviation), Proceedings of the 89th Meeting of the American Meteorological Society, Special Symposium on Weather - Air Traffic, Phoenix, AZ, 2009.

Dye, D.: Looking skyward to study ecosystem dynamics, Eos T. Am. Geophys. Un., 93, 141-143 doi:10.1029/2012EO140002, 2012.

Faugueras, O: Three-dimensional Computer Vision, MIT Press, Cambridge, ISBN 0-262-06158-9, 1993.

Feister, U. Shields, J. E.: Cloud and radiance measurements with the VIS/NIR Daylight Whole Sky Imager at Lindenberg (Germany), Meteorol. Z., 14, 627-639, doi:10.1127/0941-2948/2005/0066, 2005.

$\mathrm{Fu}$, C. L. and Cheng, H. Y.: Predicting solar irradiance with allsky image features via regression, J. Sol. Energ., 97, 537-550, doi:10.1016/j.solener.2013.09.016, 2013.

Gohari, S. M. I., Urquhart, B., Yang, H., Kurtz, B., Nguyen, D., Chow C. W., Ghonima, M., and Kleissl, J.: Comparison of solar power output forecasting performance of the Total Sky Imager and the University of California, San Diego Sky Imager, Energy Procedia, 49, 2340-2350, doi:10.1016/j.egypro.2014.03.248, 2014.

Gonzales, Y., Lopez, C., and Cuevas, E.: Automatic observation of cloudiness: analysis of all-sky images, TECO-2012, WMO Technical Conference on Meteorological and Environmental Instruments and Methods of Observation, Brussels, Belgium, 1618 October 2012.

Hartley, R. and Zisserman, A.: Multiple view geometry in computer vision, Cambridge University Press, ISBN: 9780511811685, doi:10.1017/CBO9780511811685, 2004.

Hill, R.: A lens for whole sky photographs, Q. J. Roy. Meteorol. Soc., 50, 227-235, doi:10.1002/qj.49705021110, 1924.

Huo, J. and Lu, D.: Comparison of cloud cover from all-sky imager and meteorological observer, J. Atmos. Ocean. Tech., 29, 1093 1101, doi:10.1175/JTECH-D-11-00006.1, 2012.

Johnson, R. W., Koehler, T. L., and Shields, J. E.: A Multi-Station Set of Whole Sky Imagers and A Preliminary Assessment of the
Emerging Data Base, Proceedings of the Cloud Impacts on DOD Operations and Systems - 1988 Workshop, 159-162, 1988.

Johnson, R. W., Hering, W. S., and Shields, J. E.: Automated Visibility and Cloud Cover Measurements with a Solid-State Imaging System, University of California, San Diego, Scripps Institution of Oceanography, Marine Physical Laboratory, SIO Ref. 897, GL- TR-89-0061, NTIS No. ADA216906, 1989.

Kalisch, J. and Macke, A.: Estimation of the total cloud cover with high temporal resolution and parametrization of short-term fluctuations of sea surface insolation, Meteorol. Z., 17, 603-611, doi:10.1127/0941-2948/2008/0321, 2008.

Klebe, D. I., Blatherwick, R. D., and Morris, V. R.: Ground-based all-sky mid-infrared and visible imagery for purposes of characterizing cloud properties, Atmos. Meas. Tech., 7, 637-645, doi:10.5194/amt-7-637-2014, 2014.

Long, C. N. and DeLuisi, J. J.: Development of an automated hemispheric sky imager for cloud fraction retrievals, Proceedings of the 10th Symposium on Meteorological Observations and Instrumentation, Phoenix, Arizona, American Meteorological Society, 171-174, 1998.

Long, C. N., Sabburg, J. M., Calbó, J., and Pagès, D.: Retrieving cloud characteristics from ground-based daytime color all-sky images, J. Atmos. Ocean. Technol., 23, 633-652, doi:10.1175/JTECH1875.1, 2006.

Mann, S. and Picard, R. W.: On being "undigital" with digital cameras: extending dynamic range by combining differently exposed pictures, Technical Report 323, MIT Media Lab Perceptual Computing Section, 1994 (also in: Proceedings of Imaging Science and Technology 48th annual conference, 422-428, 1995).

Marquez, R. and Coimbra, C. F. M.: Intra-hour DNI forecasting methodology based on cloud tracking image analysis, J. Sol. Energ., 91, 327-336, doi:10.1016/j.solener.2012.09.018, 2013.

Mathiesen, P. and Kleissl, J.: Evaluation of numerical weather prediction for intra-day solar forecasting in the continental United States, J. Sol. Energ., 85, 967-977, doi:10.1016/j.solener.2011.02.013, 2011.

Mathiesen, P., Collier, C., and Kleissl, J.: A high-resolution cloud-assimilating numerical weather prediction model for solar irradiance forecasting, J. Sol. Energ., 92, 47-61, doi:10.1016/j.solener.2013.02.018, 2013.

Miyamoto, K.: Fish Eye Lens, J. Opt. Soc. Am., 54, 1060-1061, doi:10.1364/JOSA.54.001060, 1964

Nakajima, T., Tonna, G., Rao, R., Boi, P., Kaufman, Y., and Holben, B.: Use of sky brightness measurements from ground for remote sensing of particulate polydispersions, J. Appl. Optics, 35, 2672 2686, doi:10.1364/AO.35.002672, 1996.

Perez, R., Cebecauer, T., and Š́ri, M.: Semi-empirical satellite models, chapter 2 in: Solar Energy Forecasting and Resource Assessment, edited by: Kleissl, J., Elsevier, ISBN: 978-0-12397177-7, 2013.

Reda, I. and Andreas, A.: Solar position algorithm for solar radiation applications, J. Sol. Energ., 76, 577-589, doi:10.1016/j.solener.2003.12.003, 2004.

Rogers, M., Miller, S., Combs, C., Benjamin, S., Alexander, C., Sengupta, M., Kleissl, J., and Mathiesen, P.: Validation and analysis of HRRR insolation forecasts using SURFRAD. Proceedings of the American Solar Energy Society, Rayleigh, NC, 2012.

Román, R., Antón, M., Cazorla, A., de Miguel, A., Olmo, F. J., Bilbao, J., and Alados-Arboledas, L.: Calibration of an all-sky 
camera for obtaining sky radiance at three wavelengths, Atmos. Meas. Tech., 5, 2013-2024, doi:10.5194/amt-5-2013-2012, 2012.

Sebag, J., Krabbendam, V. L., Claver, C. F., Andrew, J., Barr, J. D., and Klebe, D.: LSST IR camera for cloud monitoring and observation planning, Proceedings of the International Society of Photonics and Optics, Ground-based and Airborne Telescopes II conference (SPIE 7012), 7012, doi:10.1117/12.789570, 2008.

Shields, J. E., Johnson, R. W., and Karr, M. E.: Automated Whole Sky Imagers for Continuous Day and Night Cloud Field Assessment, Proceedings of the Cloud Impacts on DOD Operations and Systems Conference, 1993a.

Shields, J. E., Johnson, R. W., and Koehler, T. L.: Automated Whole Sky Imaging Systems for Cloud Field Assessment, Proceedings of the Fourth Symposium on Global Change Studies, American Meteorological Society, Boston, 17-22 January, 1993b.

Shields, J. E., Johnson, R. W., Karr, M. E., and Wertz, J. L.: Automatic day/night whole sky imager for field assessment of cloud cover distributions and radiance distributions, Proceedings of the 10th Symposium on Meteorological Observations and Instrumentations, Phoenix, AZ, American Meteorological Society, Boston, 165-170, 1998a.

Shields, J. E., Karr, M. E., Tooman, T. P., Sowle, D. H., and Moore, S. T.: The Whole Sky Imager - A Year of Progress, Proceedings of Eighth Atmospheric Radiation Measurement (ARM)Science Team Meeting, 1998b.
Shields, J. E., Karr, M. E., Johnson, R. W., and Burden, A. R.: Day/night whole sky imagers for 24-h cloud and sky assessment: history and overview, J. Appl. Optics, 52, 1605-1616, doi:10.1364/AO.52.001605, 2013.

Stumpfel, J., Tchou, C., Jones, A., Hawkins, T., Wenger, A., and Debevec, P.: Direct HDR capture of the sun and sky, AFRIGRAPH 2004, 3rd International Conference on Computer Graphics, Virtual Reality, Visualization and Interaction in Africa, Cape Town, South Africa - 3-5 November, doi:10.1145/1029949.1029977, 2004.

Urquhart, B., Ghonima, M., Nguyen, D., Kurtz, B., Chow, C. W., and Kleissl, J.: Sky imaging systems for short-term solar forecasting, chapter 9 in: Solar Energy Forecasting and Resource Assessment, edited by: Kleissl, J., Elsevier, ISBN: 978-0-12397177-7, 2013.

Urquhart, B., Kurtz, B., and Kleissl, J.: Sky camera geometric calibration using solar observations, submitted, 2015.

Wood-Bradley, P., Zapata, J., and Pye, J.: Cloud tracking with optical flow for short-term solar forecasting, Proceedings of the 50th Conference of the Australian Solar Energy Society, Melbourne, December 2012.

Yang, H., Kurtz, B., Nguyen, D., Urquhart, B., Chow, C. W., Ghonima, M., and Kleissl, J.: Solar irradiance forecasting using a ground-based sky imager developed at UC San Diego, J. Sol. Energ., 103, 503-524, doi:10.1016/j.solener.2014.02.044, 2014. 